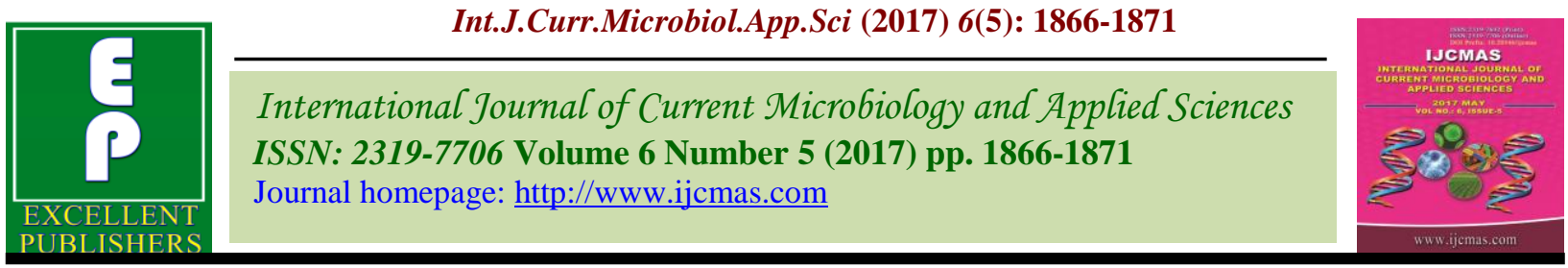

Review Article

https://doi.org/10.20546/ijcmas.2017.605.205

\title{
CRISPR/Cas9: A Nobel Approach for Genome Editing
}

\author{
Shreya $^{1 *}$, Kiran Rana ${ }^{2}$ and Ainmisha ${ }^{3}$ \\ ${ }^{1}$ Department of Genetics and Plant Breeding, IAS, BHU, Varanasi-221005, India \\ ${ }^{2}$ Department of Agronomy, IAS, BHU, Varanasi-221005 \\ ${ }^{3}$ Division of Plant Pathology, IARI, New Delhi, India \\ *Corresponding author
}

\section{A B S T R A C T}

\begin{tabular}{|l|}
\hline Ke y w o r d s \\
CRISPR/Cas9, \\
DSBs, \\
Palindromic, Guide \\
RNA, \\
Endonuclease, \\
PAM. \\
\hline Article Info \\
\hline Accepted: \\
19 April 2017 \\
Available Online: \\
10 May 2017 \\
\hline
\end{tabular}

Recently evolved technique, Clustered Regularly Interspaced Palindromic Repeats (CRISPR)-CRISPR-associated (Cas9) has added new armory for the genome editing approaches. This CRISPR/Cas9 pathway of archaeal and bacterial defense mechanism against the invading genomic material utilizes a short guide RNA to direct the endonuclease Cas9 to cut the foreign genetic material and provide resistance against the same. The immunity in archaea and bacteria is developed due to the transcription of cut segment of the exogenous material which has been incorporated in host genome system as memory which is transcribed in the form of guide RNA. So by artificially synthesizing the desired guide RNA, Cas9 can be virtually directed anywhere in the genome to cause DNA double strand breaks (DSBs) and can accomplish the repair or insertion, deletion etc actions to edit genome of the organismanlaesired directions. Theomanifestation of this novel technique depends on the presence of PAM (protospacer adjacent motif) sequence which lies downstream to the target site. Hence here we are discussing the concept and use of CRISPR/Cas9 mechanism that can be a very efficient and indispensable tool for genetic manipulation in future.

\section{Introduction}

\section{History}

In the Year 1987 marked the begin of CRISPR while studying the mechanism underlying the isozyme conversion of alkaline phosphatase in E. coli by Ishino et al., (1987) and they discovered several 'curious sequences' in the 3'end flanking region of the iap gene and described it as a set of 29 nucleotide repeats with 32 nucleotide spacing sequences.

Later short regularly repeats were reported in more than $40 \%$ of bacteria and $90 \%$ of archaea by Mojica et al., (2005). These short repeats were officially named Clustered
Repeats by Jansen et al., (2002) and the abbreviation CRISPR began to circulate widely.

Further the presence of Cas genes, situated next to CRISPR locus were identified in prokaryotes by Schouls et al., (2003). Subsequently, with the discovery of the Cas gene, Cas protein, protospacers adjacent motifs (PAM), CRISPR RNA (crRNA) and trans-activating crRNA (tracr RNA) gave the root to a genome editing mechanism. So in 2013, CRISPR/Cas mechanism of immunity in prokaryotes was established as novel 
genetic manipulation armor by Cong et al., (2013) and Mali et al., (2013).

Clustered regularly interspaced short palindromic repeats (CRISPR)-CRISPRassociated protein (Cas)9-mediated genome modification enables us to edit the genomes of a variety of organisms rapidly and efficiently. CRISPR/Cas9 is a RNA guided nuclease based genome/DNA engineering in contrast to other protein guided genome editing artificial techniques like TALENs (Transcription activator like effector nuclease) and ZFNs (Zinc finger nuclease).CRISPR/Cas was first time discovered as an acquired immune system in bacteria and archaea against foreign DNA, either viral or plasmid. The locus of CRISPR comprises of a series of conserved repeated sequences which are interspaced by unique non repetitive sequences called as spacers. During the defense mechanism in bacteria and archaea, the invading foreign DNA is cut by nuclease encoded by Cas genes and the processed small segment of invading DNA is then incorporated within the CRISPR loci as spacers in host genome. It is the spacer sequence which act as transcriptional template for producing the crRNA during the infection caused by viruses and phages. crRNA is the agent which guide the Cas to cleave the target invading sequence. There are more than 40differentCasprotein families have been reported by Haft et al., (2004) playing important roles in crRNA biogenesis, spacers incorporation and invading DNA cleavage. CRISPR/Cas system is classified into many sub classes viz., Type I, II and III based on the Cas gene phenology by Makarova et al., (2011). Only crRNA is required by Type I and III for targeting but Type II system also requires tracr RNA, Deltcheva et al., (2011). In addition there is variation in the composition of crRNA-Cas targeting complexes. Type I and III system typically consist of greater than eight subunits, Bronus et al., (2008) and Hale et al., (2009). In contrast Type II system requires only a single polypeptide, Cas9; Sapranaukas et al., (2011) which contains a HNH nuclease domain and a RuvC like nuclease domain; Jinek et al., (2012). The Cas9 is a DNA endonuclease which functions naturally via dual guide RNA (a hundred nucleotide molecule) which is constituted by fusion of a 20-nucleotide (crRNA) with a transactivating CRISPR RNA (tracrRNA); Jinek et al., (2012). The 5'end of the crRNA base pair with target DNA, but the 3' end forms a ds (double stranded) stem with the tracrRNA which thereby facilitate Cas9 nuclease recruitment. These orientations are accomplished as DNA targets are identified through RNA-DNA base pairing. Hence by making change in the sequence of the guide RNA, the targets on the DNA can be altered. One more important short sequence is also essential for the effective and efficient targeting on DNA is called as protospacer adjacent motif (PAM) which is located 3' of the protospacer element; Mojica et al., (2009). It is the PAM sequence that enables the CRISPR-Cas immune system to distinguish between the self and non-self sequence; Yosef et al., (2012). Because the PAM sequence is only present at the targets sites in the foreign DNA. Cas9 from Steptococcus pyogenes, which has been the focus of most studies to data, recognizes a 5'-NGG-3' PAM sequence; Jinek et al., 2012 and Mojica et al., (2009). Based on the complementation, the crRNA position itself at the target site on the DNA and form a RNA-DNA hetero duplex and then DNA strand of heteroduplex and its opposite stand is cleaved by the HNH nuclease domain and RuvC like domain of Cas9 and thereby generating a DSB (double stranded break) at the target site. There is always a limitation of creating double stranded break in DNA at specific sites; Carroll (2014). Methods of genome editing like TALENs and ZFNs are based on protein and the feasibility of engineering these designer enzymes to 
recognize new sequences are limited in contrast to the RNA guided genome editing through CRISPR/Cas. Also compositional simplicity of CRISPR has been paramount to its successful application. Not only does it encompass only a single polypeptide, but remarkably, it retains full activity with a chimeric single -guide (sg RNA), generated by connecting the 3 'end of crRNA to the 5'end of the tracr RNA; Jinek et al.,(2012).

\section{Genome editing with CRISPR/Cas9}

To make the genome editing and engineering process convenient, an artificial guide RNA (g RNA) is being used which contains all the attributes of crRNA and tracr RNA ; Jinek et al., (2012). Many variants of CRISPR/Cas9 has been developed to recognize 20 or 24 nucleotides sequences of engineered guide RNA and 2-4 nucleotides PAM sequence at the target site. Therefore, CRISPR/Cas9 cantheoreticallytargetaspecificDNAsequence with22-29 nucleotide which is unique in most genomes. It has been reported that the CRISPR/Cas9 is tolerant to base pair mismatch between guide RNA and its complementary target sequence; Jinek et al., (2012), Cong et al., (2013), Fu et al., (2013), Mali et al., (2013) and Hsu et al., (2013). For example, the CRISPR/Cas9 of Streptococcus pyogenes appeared to tolerate up to six base pair mismatches at target sites; Jinek et al., (2012). The non homologous end-joining (NHEJ)-mediated error-prone DNA repair and homology directed repair (HDR)mediated error-free DNA repair is carried out by DNA repair system of cell where DSB is triggered by CRISPR-Cas9 system. The NHEJ mediated DNA repair mechanism is very fast but it causes small deletion and insertion mutations at the target site thereby abolishing and disrupting the function of the target gene. INDELs were created at the yellow locus of Drosophila genome through CRISPR/Cas9-induced DNA cleavage following by NHEJ-mediated DNA repair mechanism resulted into frame shift mutation; Gratz et al., (2013). The HDR-mediated DNA repair, more complicated than NHEJmediated DNA repair. HDR-mediated errorfree DNA repair requires a homologycontaining donor DNA sequence asrepairtemplate.Throughco-injectionofCas9, two gRNA targeting, respectively, the $5^{\prime}$ and 3 ' sequences of the yellow locus, and a singlestrand oligodeoxynucleotide template, successfully replaced the yellow locus with a 50 ntattP recombination site in Drosophila genome; Gratz et al.,(2013).

\section{Advantages of CRISPR-Cas9 over other genome editing mechanisms}

There are several advantages of using this new technique of genome editing over method like TALENs and ZFNs. Being a protein guided artificial genome editing mechanism TALENs and ZFNs needs a time consuming and complicated protein engineering, selection and validation. Whereas, the CRISPR/Cas9 needs merely a short programmable guide RNA for targeting DNA and moreover the designing and production of guide RNA is relatively easy and cheap too. CRISPR/Cas9 system is efficient enough to induce genetic manipulation through repair, insertion, deletion, recombination etc. at several sites in genome independently when there is use of several guide RNA with different target sites in plants and animals; Cong et al.,(2013). Due to its simplicity this mechanism could be a useful tool to disrupt/abolish multiple genes, to investigate the gene family and to generate transgenic with multiple mutations; Wang et al., (2013) and Yang et al., (2013).

\section{Applications}

Within a few years of its discovery, CRISPR/Cas9 system has been used widely and it has reached to a wide range of hosts to target important genes of human (Mali et al., 
2013), bacteria (Fabre et al., 2014), zebra fish (Hwang et al., 2013), plants (Guo et al., 2014).

\section{Challenges}

Being a very effective, useful and easy method of genome editing, the mechanism of CRISPR-Cas9 have some serious issues regarding use of it viz., guide RNA production, delivery method of CRISPR/Cas9, dependence on PAM site and off-target mutations as well.

It is very much difficult for RNA polymerase II for synthesis gRNA due to PTMs (post translational modifications). The in vivo gRNA production is accomplished by using RNA polymerase III, U3 and U6 snRNA promoters. There is also lack of commercial availability of RNA polymerase III also limits the application of U3- and U6-based gRNA production. The delivery of the CRISPR/Cas9 into the organism is plasmid based injection techniques. More focus should be given to the delivery system to make it more efficient for different type of cells and tissues; Gratz et al., (2013). Without the PAM sequence the CRISPR/Cas9 cannot accomplish the editing process because it is the 2-5 ntPAM sequence which is required for the guide RNA to bind to the target site. Without the PAM sequence the CRISPR/Cas-9 cannot accomplish the editing process. Different Cas9 orthologs identified the different PAM sequence, such as NGG PAM from Streptococcus pyogenes; Jinek et al., (2012); Deltcheva et al., (2011), NGGNG and NNAGAAW PAM from Streptococcus thermophiles; Gasiunaset al., (2012), Garneau et al., (2010), Karvelis et al., (2013) and NNNNGATT PAM from Neisseria meningitides; Hou et al., (2013), Zhang et al., (2013). There is a high risk association of off-target mutations with the use of CRISPR/Cas-9 system of genome editing in contrast to the TALENs and ZFNs;
Fu et al., (2013). The organisms having large genome size often contain such DNA sequences that are identical or highly homologous to the target site. Under such condition CRISPR/Cas9 also cleaves non target DNA sequences resulting into off target mutations which may even cause loss in the expression of vital genes. So there much focus should be given to increase the specificity between the guide RNA and target DNA sequences to nil or minimize the off target mutation; Cong et al., (2013), Fu et al., (2013), Hsu et al., (2013) and Xiao et al., (2014).

In conclusion, CRISPR/Cas9 is an ideal genome editing tool because of its simplicity, effectiveness and versatility. Due to its user friendly nature it is gaining its popularity as one of the most potential and precise genome editing tools in the field of molecular biology. This novel technique of genome edition was first done in Drosophila melanogaster; Bibikova et al., (2002) and Bibikova et al., (2003) but due to its adaptability and success rate it has proved its power and potentials in curing the human diseases and improving the crop quality and productivity as well. In coming future CRISPR/Cas-9 system of advanced genome editing technology can be viewed as very significant genome manipulation technique in humans, animals and plants as well.

\section{References}

Bibikova, M., Golic, M., Golic, K.G. and Carroll, D. 2002.Targeted chromosomal cleavage and mutagenesis in Drosophila using zinc-finger nucleases.Genetics.Vol. 161, pp.1169-1175.

Bibikova,M., Beumer,K., Trautman,J.K., Carroll,D. 2003.Enhancing gene targeting with designed zinc finger nucleases.Science, Vol. 300, pp. 764.

Brouns, S.J., Jore, M.M., Lundgren, M., Westra, E.R., Slijkhuis, R.J., Snijders, A.P., 
Dickman, M.J., Makarova, K.S., Koonin, E.V., Van der Oost, J. 2008. Small CRISPR RNAs guide antiviral defense in prokaryotes. Science. Vol. 321, pp. 960-964.

Carroll, D. 2014. Genome engineering with targetable nucleases.Annu. Rev. Biocem. Vol. 83,pp. 409-439.

Cong, L., Ran, F.A., Cox, D., Lin, S., Barretto, R., Habib,N., Hsu,P. D., Wu, X., Jiang, W., Marraffini, L.A. 2013. Multiplex genome engineering using CRISPR/Cas systems. Science, Vol. 339, pp. 819-823.

Deltcheva, E., Chylinski, K., Sharma, C.M., Gonzales, K., Chao, Y., Pirzada, Z.A., Eckert, M.R., Vogel, J., Charpentier, E. 2011. CRISPR RNA maturation by transencoded small RNA and host factor RNase III.Nature. Vol. 471, pp. 602-607.

Fabre, L., Hello, S.L.,Roux, C., Jeanjean,S.I., and Weill, F.X. CRISPR is an Optimal Target for the Design of Specific PCR Assays for Salmonella enterica Serotypes Typhi and Paratyphi A.2014. PLoS Negl. Trop. Dis. Vol. 8:e2671.

Fu, Y., Foden, J.A., Khayter, C., Maeder, M.L., Reyon, D., Joung, J.K. and Sander, J.D. 2013. High-frequency off-target mutagenesis induced by CRISPR-Cas nucleases in human cells.Nat. Biotechnol. Vol. 31, pp. 822-826.

Garneau, J.E., Dupuis, M.E., Villion, M., Romero, D.A., Barrangou, R., Boyaval, P., Fremaux, C., Horvath, P., Magadan, A.H., Moineau, S. 2010. The CRISPR/Cas bacterial immune system cleaves bacteriophage and plasmid DNA. Nature.Vol. 468. pp. 67-71.

Gasiunas, G., Barrangou, R., Horvath, P. and Siksnys, V. 2012.Cas9-crRNA ribonucleoprotein complex mediates specific DNA cleavage for adaptive immunity in bacteria. Proc. Natl. Acad. Sci. Vol.109, pp. E2579-E2586.

Gratz, S.J., Cummings, A.M., Nguyen, J.N., Hamm, D.C., Donohue, L.K., Harrison, M.M., Wildonger, J. and O'Connor-Giles, K.M. 2013.Genome engineering of Drosophila with the CRISPR RNA- guided Cas9 nuclease.Genetics.Vol.194, pp. 1029-1035.

Guo,X., Zhang, T., Hu, Z., Zhang, Y., Shi, Z., Wang, Q., Cui, Y., Wang, F., Zhao, H., Chen, Y.Efficient RNA/Cas9mediated genome editing in Xenopus tropicalis.2014. Development. Vol. 141, pp. 707-714.

Haft, D.H., Selengut, J., Mongodin, E.F., Nelson, K. E. 2005. A guild of 45 CRISPR-associated (Cas) protein families and multiple CRISPR/Cas sub types exist in prokaryotic genomes. PLoSComput. Biol. Vol. 1, pp. 60.

Hale, C.R., Zhao, P., Olson, S., Duff, M.O., Graveley, B.R., Wells, L., Terns, R.M., Terns, M,P. 2009. RNA-guided RNA cleavage by a CRISPR RNA-Cas protein complex.Cell. 2009.Vol. 139, pp. 945956.

Hou, Z., Zhang, Y., Propson, N.E., Howden, S.E., Chu, L.F., Sontheimer, E.J. and Thomson, J.A. 2013. Efficient genome engineering in human pluripotent stem cells using Cas9 from Neisseria meningitidis.Proc. Natl. Acad. Sci. USA. Vol. 110, pp. 15644-15649.

Hsu, P.D., Scott, D.A., Weinstein, J.A., Ran, F.A., Konermann, S., Agarwala, V., Li, Y., Fine, E.J., Wu, X., Shalem, O. 2013. DNA targeting specificity of RNA-guided Cas9 nucleases.Nat. Biotechnol. Vol. 31, pp. 827-832.

Hwang, W.Y., Fu, Y., Reyon, D., Maeder, M.L., Tsai, S.Q., Sander, J.D., Peterson, R.T., Yeh, J.R., Joung, J.K. 2013. Efficient genome editing in zebrafish using a CRISPRCas system.Nat Biotechnol. Vol.31, pp. 227-229.

Ishino Y., Shinagawa, H., Makino, K., Amemura, M., Nakata, A. 1987. Nucleotide sequence of the iap gene, responsible for alkaline phosphatase isozyme conversion in Escherichia coli, and identification of the gene product. $J$ Bacteriol.Vol.169, pp. 5429-5433.

Jansen, R., Embden, J.D., Gaastra, W., Schouls, L.M. 2002. Identification of genes that are associated with DNA repeats in 
prokaryotes. MolMicrobiol. Vol. 43, pp. 1565-1575.

Jinek, M., Chylinski, K., Fonfara, I., Hauer, M., Doudna, J.A., Charpentier, E. 2012. A programmable dual-RNA-guided DNA endonuclease in adaptive bacterial immunity. Science. Vol. 337, pp. 816821.

Karvelis, T., Gasiunas, G., Miksys, A., Barrangou, R., Horvath, P., Siksnys, V. 2013. crRNA and tracrRNA guide Cas9mediated DNA interference in Streptococcus thermophilus. RNA Biol. Vol. 10. pp. 841-851.

Mali, P., Aach, J., Stranges, P.B., Esvelt, K. M., Moosburner, M., Kosuri, S., Yang, L., Church, G. M. 2013.CAS9 transcriptional activators for target specificity screening and paired nickases for cooperative genome engineering.Nat. Biotechnol. Vol. 31, pp. 833-838.

Makarova, K.S., Haft, D.H., Barrangou, R., Brouns, S.J., Charpentier, E., Horvath,P.,Moineau,S.,Mojica,F.J.,Wolf, Y.I.,Yakunin,A.F. 2011.Evolution and classification of the CRISPR-Cas systems.Nat. Rev. Microbiol. Vol. 9, pp. 467-477.

Mojica FJ, Diez-Villasenor C, Garcia-Martinez J andSoria E. 2005. Intervening sequences of regularly spaced prokaryotic repeats derive from foreign genetic elements. J MolEvol.Vol. 60, pp. 174 182.

Mojica, F.J.M., Diez-Villaserior,G., GarciaMartinez, J., Almendros, C. 2009. Short motif sequences determine the targets of the prokaryotic CRISPR defence system. Microbiology. Vol. 155, pp. 733-740.

Schouls, L. M., Reulen, S., Duim, B., Wagenaar, J.A., Willems, R.J., Dingle, K.E., Colles, F.M., Van Embden, J.D.
2003. Comparative genotyping of Campylobacter jejuni by amplified fragment length polymorphism, multilocus sequence typing, and short repeat sequencing: strain diversity, host range, and recombination. $J$ ClinMicrobiol.Vol. 41, pp. 15 -26.

Sapranauskas, R., Gasiunas, G., Fremaux, C.,Barrangou, R., Horvath, P., Siksnys, V. 2011. The Streptococcus thermophilus CRISPR/Cas system provides immunity in Escherichia coli. Nucleic Acids Res. Vol. 39, pp. 9275-9282.

Wang, H., Yang, H., Shivalila, C.S., Dawlaty, M.M., Cheng, A.W., Zhang, F., Jaenisch, R. 2013.One-step generation of mice carrying mutations in multiple genes by CRISPR/Cas-mediated genome engineering.Cell.Vol. 153. pp. 910-918.

Xiao,A., Cheng, Z., Kong, L., Zhu,Z., Lin,S., Gao,G., Zhang,B. 2014. CasOT: a genome-wide Cas9/gRNA off-target searching tool. Bioinformatics.[Epub ahead of print].

Yang, H., Wang, H., Shivalila, C.S., Cheng, A.W., Shi, L., Jaenisch, R. 2013.One-step generation of mice carrying reporter and conditional alleles by CRISPR/Casmediated genome engineering. Cell.Vol.154, pp. 1370-1379.

Yosef, I., Goren, M.G., Qimron, U. 2012. Proteins and DNA elements essential for the CRISPR adaption process in Escherichia coli. Nucleic Acids Res. Vol. 40, pp. 5569-5576.

Zhang, Y., Heidrich, N., Ampattu, B.J., Gunderson, C.W., Seifert, H.S., Schoen, C., Vogel, J. and Sontheimer, E.J. 2013. Processing-independent CRISPR RNAs limit natural transformation in Neisseria meningitidis. Mol. Cell. Vol.50,pp.488503.

\section{How to cite this article:}

Shreya, Kiran Rana and Ainmisha. 2017. CRISPR/Cas9: A Nobel Approach for Genome Editing. Int.J.Curr.Microbiol.App.Sci. $\quad 6(5): \quad 1866-1871 . \quad$ doi: https://doi.org/10.20546/ijcmas.2017.605.205 